\title{
Correction to "Various Structures Associated to the Representation Categories of Eight-Dimensional Non-Semisimple Hopf Algebras" [Algebras and Representation Theory 7, 491-515, (2004)]
}

\section{Michihisa Wakui}

Published online: 16 January 2009

(C) Springer Science + Business Media B.V. 2009

There are some errors on determination of ribbon elements in the above article. The statements and the proofs on determination of ribbon elements in Proposition 2.7 and Proposition 2.9 are wrong. A correct version of the statements of Proposition 2.7 and Proposition 2.9 is as follows (all corrections are indicated by shaded area):

Proposition 2.7' Let $\boldsymbol{k}$ be an algebraically closed field of characteristic 0. Then a universal $R$-matrix of $A_{C_{2} \times C_{2}}$ is given by

$$
R_{1000}(1 \otimes 1+a x \otimes g x) \text { or } R_{0111}(1 \otimes 1+a x \otimes g x),
$$

where $a \in \boldsymbol{k}$, and $R_{1000}, R_{0111}$ are those universal $R$-matrices of $\boldsymbol{k}\left[C_{2} \times C_{2}\right]$ which appear in Lemma 2.3(2). Any quasitriangular Hopf algebra $\left(A_{C_{2} \times C_{2}}, R\right)$ has exactly two ribbon elements, which are given by 1 and gh.

Proposition 2.9' (Gelaki) Let $\boldsymbol{k}$ be an algebraically closed field of characteristic 0. Then the universal $R$-matrices of $A_{C_{2}}$ are given by

$$
\begin{aligned}
R= & \sum_{\alpha, \beta=0,1}(-1)^{\alpha \beta}(1 \otimes 1-(a d-b c) x y \otimes x y)\left(E_{\alpha} \otimes E_{\beta}\right) \\
& +\sum_{\alpha, \beta=0,1}(-1)^{\alpha(\beta+1)}(a x \otimes x+b x \otimes y+c y \otimes x+d y \otimes y)\left(E_{\alpha} \otimes E_{\beta}\right),
\end{aligned}
$$

The online version of the original article can be found at http://10.1023/B:ALGE.0000048318.64013.db.

M. Wakui $(\varangle)$

Department of Mathematics, Faculty of Engineering Science, Kansai University,

Suita-shi, Osaka 564-8680, Japan

e-mail: wakui@ipcku.kansai-u.ac.jp 
Table 3

\begin{tabular}{llll}
\hline Hopf algebras & $\begin{array}{l}\text { The universal } \\
R \text {-matrices }\end{array}$ & The ribbon elements & Remarks \\
\hline$A_{C_{4}}^{\prime}$ & Do not exist & Do not exist & \\
$A_{C_{4}}^{\prime \prime}$ & Do not exist & Do not exist & Triangular \\
$A_{C_{4, \omega}^{\prime \prime \prime}}$ & $R_{2}$ (unique) & 1 & Triangular \\
$A_{C_{2} \times C_{2}}$ & $R_{q}(1 \otimes 1+a x \otimes g x)$ & $1, g h$ & Triangular \\
$\left(A_{C_{4}}^{\prime \prime}\right)^{*}$ & $(a \in \boldsymbol{k}, q=0,1)$ & & Not triangular \\
$A_{C_{2}}($ unique $)$ & 1 & (if $b \neq c)$ \\
\hline
\end{tabular}

where $a, b, c, d \in \boldsymbol{k}$ are arbitrary. The quasitriangular Hopf algebra $\left(A_{C_{2}}, R\right)$ has exactly one ribbon element, which is given by $1+(c-b) x y$.

Remark The same statement as Proposition 2.9' has already appeared in the Gelaki's paper [1, Proposition 2.2.3], although we cited Proposition 2.9 as a result of him in our published paper. The misdescription on ribbon elements in Proposition 2.9 comes from my carelessness.

With these corrections, the ribbon elements for the Hopf algebras $A_{C_{2} \times C_{2}}$ and $A_{C_{2}}$ listed in the Table 3 on the page 495 should be also changed as follows:

In the proof of Proposition 2.7, on the page 501, the eighth line from the bottom is wrong. It should be correctly replaced by "If a central element $v \in A_{C_{2} \times C_{2}}$ satisfies $\varepsilon(v)=1$ and $v^{2}=u S(u)$, then $v=1$ or $v=g h$." In the proof of Proposition 2.9, on the page 504, the seventh line from the bottom is wrong. It should be correctly removed " $\Longleftrightarrow b=c$," and the next sentence should be correctly replaced by "Therefore, the above $v$ is a ribbon element of $\left(A_{C_{2}}, R\right)$."

We have another proof for the results on ribbon elements in Proposition $2.7^{\prime}$ and Proposition $2.9^{\prime}$. Actually, they are quite easily shown by using the following fact. For an element $v \in A$ in a quasitriangular Hopf algebra $(A, R)$ over a field $\boldsymbol{k}$, the following are equivalent (for example, see [2, p.763-764]):

i) $v$ is a ribbon element,

ii) there exists a group-like element $g$ such that $v=g^{-1} u$ is in the center of $A$ and $S(u)=g^{-2} u$, where $u \in A$ is the Drinfeld element of $(A, R)$.

For the quasitriangular Hopf algebra $\left(A_{C_{2}}, \bar{R}_{a b c d}\right)$, the Drinfeld element is given by $u=g+(c-b) g x y$, which satisfies $S(u)=u$, and $G\left(A_{C_{2}}\right)=\{1, g\}$. Hence, the candidates of ribbon elements are $1^{-1} u=u$ and $g^{-1} u=1+(c-b) x y$. Since $1+(c-$ $b$ ) $x y$ belongs to the center of $A_{C_{2}}$ whereas $u$ dose not, $1+(c-b) x y$ is the unique ribbon element of $\left(A_{C_{2}}, \bar{R}_{a b c d}\right)$. 
A similar argument is also applicable to any quasitriangular Hopf algebra $\left(A_{C_{2} \times C_{2}}, R\right)$, and we see that it has exactly two ribbon elements, which are given by 1 and $g h$.

\section{References}

1. Gelaki, S.: On pointed ribbon Hopf algebras. J. Algebra 181, 760-786 (1996)

2. Kauffman, L.H.: Gauss codes, quantum groups and ribbon Hopf algebras. Rev. Math. Phys. 5, 735-773 (1993) 